Open Communications in Nonlinear Mathematical Physics $]$ OCnmp $\quad$ Vol.1 (2021) pp 20-40 ARTICLE

\title{
Vaccination strategies for a seasonal epidemic: a simple SIR model
}

\author{
G. Nakamura, B. Grammaticos and M. Badoual \\ Université Paris-Saclay, CNRS/IN2P3, IJCLab, 91405 Orsay, France and Université de \\ Paris, IJCLab, 91405 Orsay France
}

Received 6 May 2021; Accepted 29 May 2021

\begin{abstract}
We model the effect of vaccination on an epidemic which, like the current one, exhibits a climate-induced seasonality. Our study is carried out using a simple SIR model. One important feature of our approach is that of recruitment: by gradually introducing susceptible individuals we mimic the spatial evolution of the epidemic, which is absent in the classic SIR.

We focus on the effect of vaccination on the number of hospital admissions. We show that any delay in the vaccination campaign results in an increase of hospitalisations, and if one tries to palliate for the delay by increasing the vaccination rate, this results in an inflation of the number of necessary doses. Considering a multi-agegroup population we show that it is advantageous to prioritise the vaccination of the older groups (upholding thus the current practice). Finally, we investigate whether a vaccination of the younger population based on awareness can be an optimal strategy, concluding by a negative.
\end{abstract}

\section{Introduction}

Mathematical modelling in the case of virulent epidemics has a particular interest [1]. On the one hand it allows to explore a slew of scenarios, the implementation of which cannot be considered for practical or ethical reasons. This may lead to specific recommendations allowing the decision makers to reach properly informed decisions. On the other hand, a careful analysis of the results of the models can yield novel insights into the mechanism of the epidemic, providing useful feedback for the refinement of the model itself as well as the associated scenarios.

In the case of the COVID-19 pandemic, the main objective is to seek the best approach(es) to mitigate the epidemic. Despite the ongoing progress in planning effective treatments for infected individuals and developing efficient drugs, the best route remains that of prevention. The way to implement the latter is two-pronged. The first is through the various non-pharmaceutical interventions (NPI), consisting in a series of restrictions and recommendations. The second is through a large-scale vaccination campaign. The

(C) The author(s). Distributed under a Creative Commons Attribution 4.0 International License 
latter approach has been made possible thanks to the development and production, in record time, of efficient vaccines which, at least according to the results existing to date, can prevent infections from the SARS-CoV-2 virus.

The question of NPI has been the object of a profusion of publications. A usual approach is to analyse data obtained over a more or less extended region with the help of some model and formulate predictions, and, in some cases, recommendations. N. Perra, in his review article [2], presents a bibliography of close to 400 articles. He points out that, while everybody agrees that infectious diseases and human behaviour are intertwined, our understanding of the feedback loop was limited till the appearance of COVID-19, which is a real game-changer in this domain. Several governments $[3,4]$ have set up panels which monitor the effect of the NPI not only locally but in a host of countries and subnational jurisdictions. Brauner et al. [5] analysed the effectiveness of governmental NPI on the COVID-19 epidemic in a selection of countries, reaching the, somewhat disappointing, conclusion that not all interventions were equally effective. This resonates with the message of the ECDC, highlighted in their "Guidelines for the implementation of non-pharmaceutical interventions against COVID-19" [6]. While conceding that the NPI have played a critical role in reducing transmission rates and the impact of COVID-19 in Europe, they acknowledge that most NPI can have a negative impact on the general well-being of people, the functioning of society, and the economy. Their conclusion is that the use of NPI is more effective when tailored to the local epidemiological situation. Our work [7] is in line with this recommendation. Referring to the 2020 epidemic situation and the lockdown policy of the French government, we studied the various confinement-exit strategies, aiming at minimising the severity of a second epidemic wave which usually accompanies the relaxation of social distancing measures. A similar study was conducted by Fokas and collaborators [8] targeting the lockdown exit strategies in Greece. They introduced a two-subpopulation model, comprising 'young' and 'old' individuals, concluding that it is preferable to ease the lockdown measures for the young subpopulation rather than for the older one.

In this paper we shall not deal with the question of the impact of NPI but rather focus on the importance of vaccinations. The question of vaccination and its effect on epidemics is not a new one. One can even trace it back to what is probably the first paper introducing a mathematical approach to epidemics [9]. Closer to us, Feng and collaborators [10] investigated the effect of vaccinations on an influenza pandemic. Just before the emergence of the COVID-19 epidemic, Widyaningsih et al. [11] studied the effect of vaccination on tuberculosis transmission in Indonesia. A vast collaboration within the Vaccine Impact Modelling Consortium [12] addressed the question of the impact on health of vaccination against a collection of pathogens. They concluded, on the basis of the results of their model, that millions of deaths may be averted, compared to the (admittedly counterfactual) scenario of non-vaccination.

In the case of COVID-19, which is the one we are interested in, the efficiency of existing vaccines has been established by several clinical studies $[13,14]$. But what is even more compelling are the real-life, large-scale, results obtained from the massive vaccination campaign in Israel. Chodick and collaborators [15] analysed data from half a million vaccinated persons and concluded on the effectiveness of the vaccine already two-three weeks after the first dose, a second dose being however necessary in order to attain the anticipated protection. Based on the above study, on that of Hunter et al [16], and on a collection of data from the Israel Ministry of Health, Mahase [17] concluded epigrammat- 
ically that "Israel sees new infections plummet following vaccination". In fact the data from Israel show that the infection probability is smaller than 1 per thousand of fully vaccinated individuals. But even this estimate sounds rather cautious. The recent data of the CDC, based on more than 100 million vaccinations in the US, reported [18] around 10 thousand breakthrough infections, corresponding to an order of magnitude larger vaccine efficiency compared to that obtained from the Israel data.

In what follows we shall explore the effects of vaccination following various implementation scenarios in the framework of a simple SIR model.

\section{The model}

The model we shall use in order to assess the effects of vaccination is none other than the classical SIR model. Introduced by Kermack and McKendrick [19], inspired by the works of Ross [20], the model is the prototypical compartmental model for epidemics. It considers a population spilt into three parts: the 'healthy' individuals that are susceptible to infection $S$, the 'infective' ones who can transmit the disease $I$, and the 'removed' $R$ who either died from the disease or, having recovered, are immune to it. This latter point is an important assumption as we shall see in what follows. The differential equations governing the SIR model are

$$
\begin{gathered}
\frac{d S}{d t}=-a S I \\
\frac{d I}{d t}=a S I-\lambda I \\
\frac{d R}{d t}=\lambda I,
\end{gathered}
$$

with $a$ being the infection rate and $\lambda$ the removal rate of the infected individuals. The interaction term, $S I$, stems from the assumption that the number of infected individuals increases at a rate proportional to the number of both infected and healthy. Given the form of (1) it follows that the total population $S+I+R$ is constant. Assuming that $S$, $I$ and $R$ are fractions of the population, we can normalise this constant to 1 . The ratio $a / \lambda$ defines what is called the basic reproduction number i.e. the expected number of infections in the susceptible population resulting from a single infection. This is usually referred to as $R_{0}$. The parameter $\lambda$ fixes the time scale. In the case of the COVID-19 epidemic, we shall be focusing on in this paper, the value of $\lambda$ is 5 days, as we argued in $[7]$.

In [21] we presented our arguments in favour of the use of the SIR rather than the SEIR model, which one usually encounters in covid-related studies. Following the findings of Aleta and Merano [22] we have also found that the correlation between the results of the two models is perfect. The main effect of the presence of the extra, "exposed", component in SEIR being a delay in the growth of the epidemic compared to the SIR results. A further argument is based on the work of Roda and collaborators [23] who, using the Akaike information criterion for model selection, argued that the SIR model performs better than the SEIR in representing the information contained in the confirmed-case data. 
The existing epidemic data, covering more than a year are compatible with a seasonal behaviour of the epidemic. Already laboratory studies [24], [25] of the effect of ultraviolet radiation on the virus had concluded on a possible seasonality. Moreover the existence of a correlation between the geographic latitude and the transmission of the virus, observed by Sajadi and collaborators [26] is consistent with the behaviour of a seasonal respiratory virus. The argument in favour of seasonality is reinforced by the findings of Watanabe [27] who compared the evolution of the epidemic over the first few months of the year in the north and south hemispheres. The differences between the two regions are striking and since Watanabe took extra care to eliminate other possible explanations of the observed behaviour, one can conclude that there exists a clear statistical evidence in favour of seasonality. As a consequence of this, we are led to extend the model given in (1), introducing a time-dependence in the parameter $a$. In particular we assume that $a(t)$ varies periodically around some mean value with a period corresponding to one year [10]. This can be represented by a simple sinusoidal expression $a(t)=\alpha+\beta \cos (2 \pi t / T)$ where $T$ stands for the duration of a year. The phase of the cosine chosen corresponds to the maximum of $a(t)$ occurring at the beginning of the year, representing the situation in the northern hemisphere. A phase shift of $T / 2$ would be necessary in the case of the southern hemisphere. It is clear that the simple cosine representation of the seasonality effect may appear as rather naïve. However it has the advantage of being extremely simple, allowing one to visualize the effect without complicating the calculations.

Before introducing vaccination it is important to discuss the question of immunity. Several studies have addressed this question. Dan et al [28] concluded that substantial immunological memory is generated after a COVID-19 infection. Gaebler et al [29] found that individuals who had been infected with the SARS-CoV-2 virus could mount a rapid and effective response to the virus upon re-exposure. If one eschews the possibility of infection of vaccinated individuals, based on the more than encouraging results of the vaccination campaign in Israel, United Kingdom and the US of A, one obtains the model:

$$
\begin{gathered}
\frac{d S}{d t}=-a(t) S I-v \\
\frac{d I}{d t}=a(t) S I-\lambda I \\
\frac{d R}{d t}=\lambda I+v .
\end{gathered}
$$

(A similar model was considered by Wong et al. [30] in their study of the vaccination campaign program in Malaysia, albeit without any seasonality effect). The vaccination term $v$, such as given in (2), corresponds to a constant-rate vaccination. This is not an essential constraint. The rate can be easily made time-dependent and/or dependent on the other components of the model, like the number of infected individuals. We shall come back to this point later. One tacit assumption of (2) is that there is no an a priori vaccine mistrust [31]: the susceptible population gets vaccinated once vaccines become available. One subtle problem, which is essentially ignored in our approach, is the fact that in several countries the adopted strategy is to vaccinate also people who have been infected and who have recovered. To the latter one must add those who have been infected and, having had no sysmptoms, did not realise that they went through the infection and get vaccinated just like the ones who had not been infected. The result of this is that the removal rate due to 
vaccination is lower than the one grossly estimated. As far as our model is concerned this is not a real problem, since we do not allow re-infections. For us: 'once removed, always removed'. But the vaccination of already infected individuals could play a role if one were to consider a finer structure of the population.

Our initial assumption is that the vaccine efficiency is perfect and the results presented in subsection 3.1 will be based on this assumption. However, and in order to set our minds in peace, we must also study the effect of a vaccination that confers incomplete immunity. This question will be addressed in subsection 3.2.

Having set the frame, we proceed now to prepare the model equations for simulation. In order to do this, it is necessary to produce a discrete version of system (2). At this point it is interesting to point out that Kermack and McKendrick [19] established the eponymous model starting from a set of discrete equations. Of course, these equations were meant as a guide for the construction of the continuous system and were not meant as an integrator thereof. Our discretisation approach has been summarised in various publications [32], [33]. It follows the ideas of Mickens [34] and uses the fact that the quantities appearing in the SIR model are by definition positive. (As shown in [35] the positivity of the solution of SIR is guaranteed provided one starts from positive initial conditions). The aphorism-like prescription we proposed in [32] states that 'if all quantities are positive, no minus sign should appear anywhere'. In order to discretise (2) we introduce a forward difference of the time derivative, with time step $\delta$, and, what is more important, a specific staggering. Unfortunately this is not sufficient for the disappearance of minus signs, due to the presence of the vaccination term. Fortunately the same difficulty was encountered in [32] where we proposed a simple solution. It suffices to replace the $-v$ term by $-v S / S$ and introduce the staggering $-v S_{n+1} / S_{n}$. This allows us to obtain the discrete system

$$
\begin{gathered}
\frac{S_{n+1}-S_{n}}{\delta}=-a_{n} I_{n} S_{n+1}-v \frac{S_{n+1}}{S_{n}} \\
\frac{I_{n+1}-I_{n}}{\delta}=a_{n} I_{n} S_{n+1}-\lambda I_{n+1} . \\
\frac{R_{n+1}-R_{n}}{\delta}=\lambda I_{n+1}+v \frac{S_{n+1}}{S_{n}} .
\end{gathered}
$$

Solving for the points at $(n+1)$ we obtain

$$
\begin{gathered}
S_{n+1}=\frac{S_{n}}{1+a_{n} \delta I_{n}+v \delta / S_{n}} \\
I_{n+1}=\frac{I_{n}\left(1+a_{n} \delta S_{n+1}\right)}{1+\lambda \delta} \\
R_{n+1}=R_{n}+\lambda \delta I_{n+1}+v \delta \frac{S_{n+1}}{S_{n}} .
\end{gathered}
$$

Given the structure of (4) it is clear that $S_{n}+I_{n}+R_{n}$ is a constant and just as in the differential case, since we shall work with fractions of the population, we normalise it to 1 . Note that the introduction of the $S_{n+1} / S_{n}$ term has as a consequence that the equations are no more time-reversible. However this is not of particular importance since we are interested in the forward-in-time evolution of the epidemic. 
While the SIR model has the advantage of great simplicity, it also has a serious drawback, that of lack of spatial dependence. The implicit assumption in system (1) is that the total population is concentrated on a single point. However it is clear that an epidemic exhibits a rich spatial structure [36]. The situation is further complicated by the fact that travellers may introduce the disease into heretofore infection-free areas [37]. A necessity for epidemic-spreading models was already present before the emergence of the COVID-19 epidemic. Thus Saito et al [38] used a multi-centre model, based on SEIR, in order to describe the 2009, H1N1 influenza in Japan. Similarly Zhao et al [39] studied the effect of population recruitment on the HIV epidemic. An application to the COVID-19 case can be found in the work of Cooper and collaborators [40]. They investigate the spreading of COVID-19 in a SIR framework, one where the susceptible population is taken as a variable to be adjusted at various times in order to account for the infection spreading. Practically, this is done by introducing surges in the susceptible population whenever the authors deem this necessary. In [21] we introduced our own approach to population recruitment. Starting from the discrete system (4) we add to $S_{n+1}$ obtained from the first equation a quantity $\Delta_{n}$. And we normalise all three quantities by introducing

$$
\begin{aligned}
\tilde{S}_{n} & =\frac{S_{n}+\Delta_{n}}{1+\Delta_{n}} \\
\tilde{I}_{n} & =\frac{I_{n}}{1+\Delta_{n}} \\
\tilde{R}_{n} & =\frac{R_{n}}{1+\Delta_{n}} .
\end{aligned}
$$

It is clear that, thanks to this normalisation, we have $\tilde{S}_{n}+\tilde{I}_{n}+\tilde{R}_{n}=S_{n}+I_{n}+R_{n}=1$. In the aforementioned paper we considered various recruitment patterns in order to reproduce the evolution of the epidemic observed in a selection of countries. In this paper we are going to stick to a very simple recruitment scenario. We shall study the vaccination effect on an epidemic which exhibits seasonality, waxing during winter, waning during summer and reappearing again during the autumn period. The estival habits in most European countries are such that a non-negligible part of the population travels to the preferential destinations of vacationers. This local increase of the susceptible population has as a consequence the acceleration and intensification of the epidemic resurgence. Our recruitment function will be a simple constant $\Delta$, non-zero over two summer months, in the case of the northern hemisphere. While this sounds perfectly reasonable, it is clear that massive vaccinations, like the ones practiced in most European (but not only) countries, would perturb our recruitment assumption, which should now be interpreted as concerning the non-vaccinated travelling individuals.

The recruitment model presented in (5) may appear somewhat opaque to people who are not familiar with discrete systems. In order to make things transparent we present the same model in a continuous (differential) setting. Assuming a recruitment rate $\mu(t)$ we find that equation (1) becomes

$$
\begin{gathered}
\frac{d S}{d t}=-a S I+\mu(t)(1-S) \\
\frac{d I}{d t}=a S I-\lambda I-\mu(t) I
\end{gathered}
$$




$$
\frac{d R}{d t}=\lambda I-\mu(t) R
$$

Given the form of (6) it is clear that if we start with $S+I+R=1$ the recruitment effect does not modify this constant of motion. In what follows, the vacation-associated recruitment $\mu(t)$ is a step function : $\mu=0$ for $t<7$ months, $\mu=\mu_{0}$ for $7<t<9$ months, and $\mu=0$ for $t>9$ months.

\section{Results for a single component population}

In this section we shall present results obtained from simulations using our model in the case where the population is considered as a homogeneous one, without any age-based stratification. We believe that this drastic simplification is most useful in order to highlight the main features of the model and their influence on the epidemic.

\subsection{The case of a perfectly efficient vaccine}

We start with the seasonality assumption and investigate the persistence of the epidemic over successive years. An example of such a situation is presented in Figure 1. The reproduction number varies with time, because of seasonality: $R_{0}(t)=a(t) / \lambda$ and $a(t)=$ $a_{0}(1.0+0.25 \cos (2 \pi t / T))$, with $a_{0}=1.2$ and $T=12$ months.

The choice of the model parameters in Figure 1 was such that the epidemic disappeared after a few years. However it is equally possible, with the proper choice of parameters to reach an endemic situation, where the epidemic is recurring, year after year. A vaccination campaign can have a significant influence of the evolution of the epidemic, making it disappear much earlier than in the case where it was let to run its course, see Figure 1.

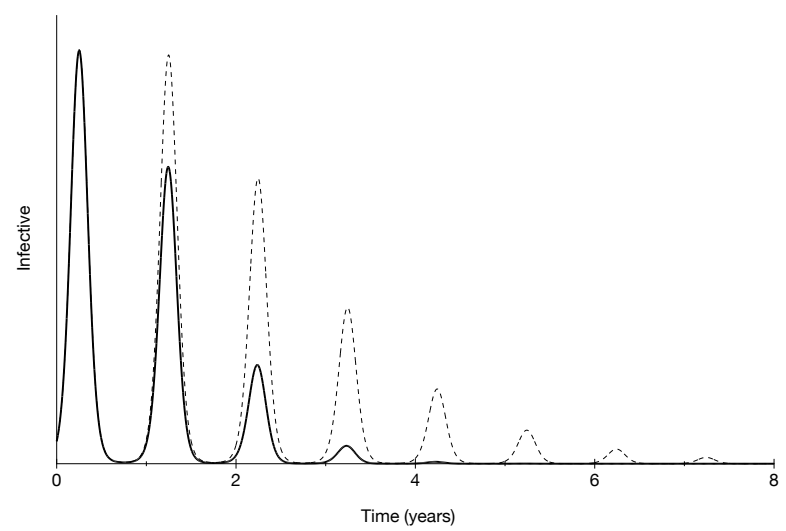

Figure 1. Temporal evolution of the infective fraction of the population over the years, without vaccination (dashed line) and with vaccination (solid line, $v / \lambda=2.510^{-3}$ ). The initial infective population is $I(0)=1.510^{-5}$. Vaccination starts at $t=2$ months.

In what follows we shall not pay further attention to the question of the endemic or not character of the epidemic but focus on the situation where the epidemic recurs after a first bout. In particular we shall investigate the effect of vaccination on the epidemic as a whole but more specifically on the second peak, see Figure 2. For all the figures presented below the simulation starts with $I(0)=6.810^{-6}, R(0)=0$ and $S(0)=1-I(0)$. 


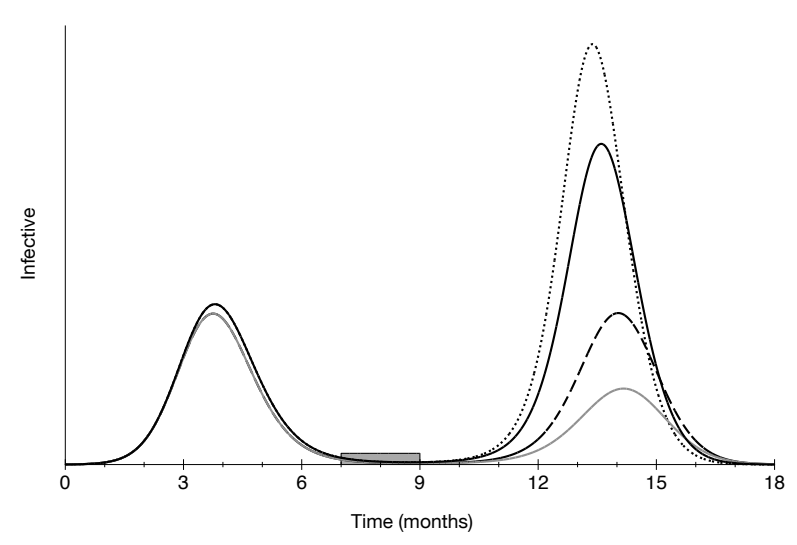

Figure 2. Temporal evolution of the infective fraction of the population, without vaccination (solid black line), with vaccination (solid grey line), with recruitment but without vaccination (dotted line), with recruitment and with vaccination (dashed line). When present, vaccination starts at $t=2$ months and $v / \lambda=2.510^{-3}$. Here, $\mu_{0} / \lambda=1.2810^{-4}$.

As explained in the caption of Figure 2, four different scenarios are presented. We have namely the profile of the epidemic (as given by the infective population) under the influence of seasonality, with and without vaccination, and including or not the recruitment effect due to vacations. We have chosen the infection rate so as to have, in the case without vaccination, a second peak twice as large as the first one. The vaccination rate was adjusted so as to make the second peak half as large as the first. The vacation recruitment rate was fixed so as to make the second epidemic peak, under vaccination, equal to the first.

It is interesting at this point to show the effect of vaccinations as well as of the vacationassociated recruitment on the susceptible population.

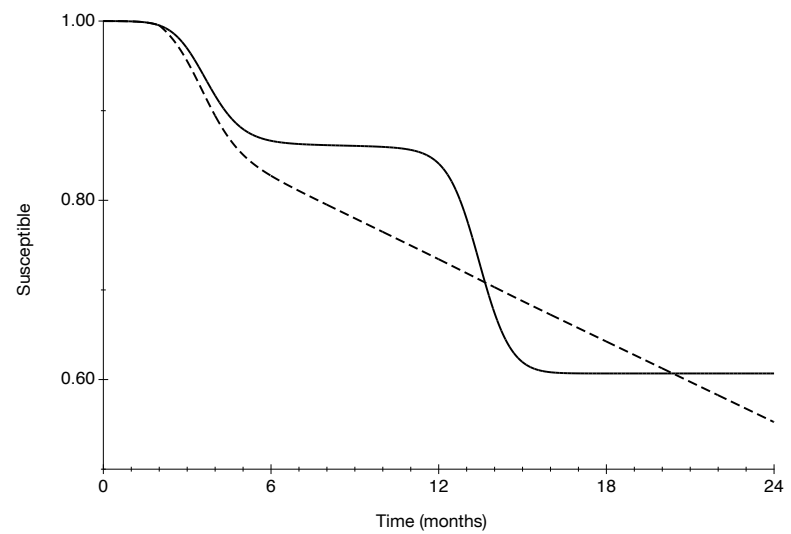

Figure 3. Temporal evolution of the susceptible fraction of the population, without (solid line) and with (dashed line) vaccination. When present, vaccination starts at $t=2$ months and $v / \lambda=2.510^{-3}$.

Figure 3 compares the evolution of the susceptible population with and without vaccination. While in the case without vaccination we are in the presence of two epidemic waves, the effect of the vaccination campaign is to wash out completely the second one. 


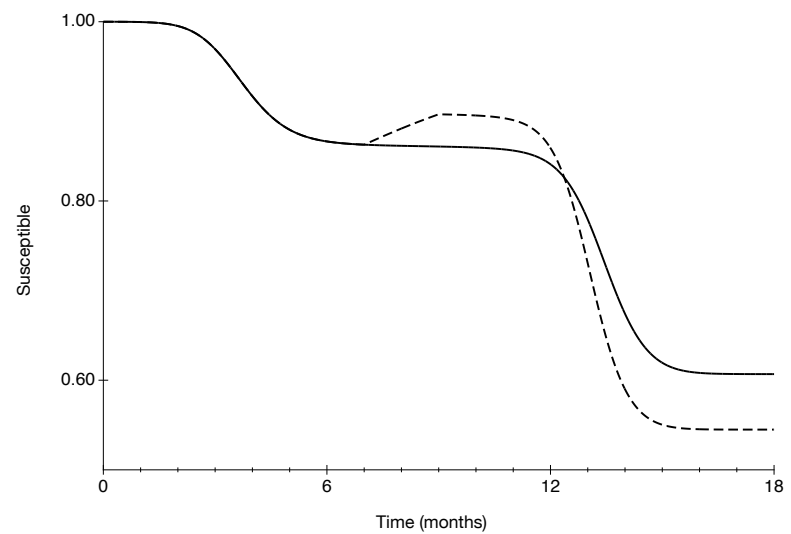

Figure 4. Temporal evolution of the susceptible fraction of the population, without (solid line) and with (dashed line) recruitment. For recruitment, $\mu_{0} / \lambda=1.2810^{-4}$.

The recruitment during the vacation period is shown in Figure 4. The recruitmentinduced increase of susceptible population has as a consequence not only a stronger second epidemic wave (as can be seen in Figure 2) but also an additional depletion of the susceptible population as well as a slightly hastened appearance of said wave.

Having shown how our model works we proceed now to use it in order to try to answer a selection of pertinent questions. The first such question is what does happen if one delays vaccination so that it does not start at the second month as was done in the simulation presented in Figure 2.

In order to account for the effect of vaccination, we need to introduce the number of hospitalisations. The effect of vaccination is to reduce the number of susceptible, see equations (2). When vaccinated, these people go directly to the "removed" compartment. In the following, the total number of hospitalisations is proportional to the total number of "removed" that have not been vaccinated.

In the case of delayed vaccination, one can hope to compensate for the delay in the beginning of vaccinations by increasing the vaccination rate so as to keep a ratio of $1 / 2$ between the maximum numbers of infected in the first and second wave.

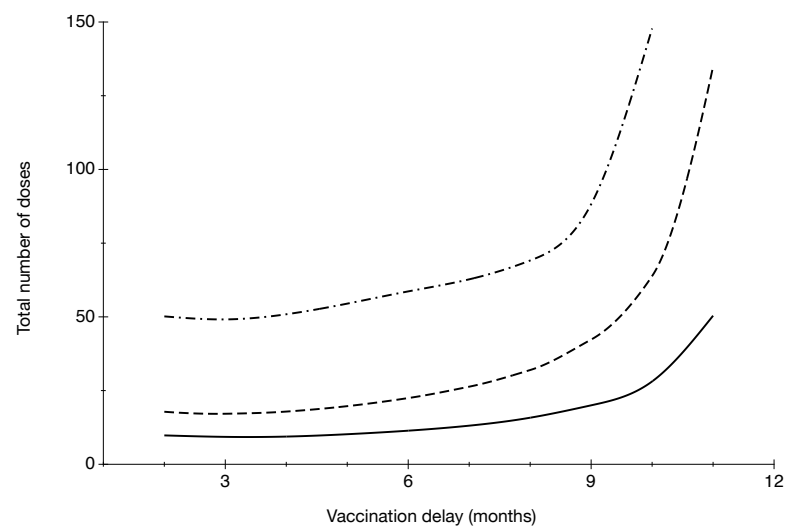

Figure 5. Number of vaccine doses necessary to keep the peak of the second wave of hospitalisations at a value half of that of the one of the first wave, as a function of the vaccination delay, without recruitment (solid line), with a vacation-associated recruitment 
$\left(\mu_{0} / \lambda=10^{-4}\right.$, dashed line $)$, and an even stronger recruitment $\left(\mu_{0} / \lambda=10^{-3}\right.$, dashed-dotted line).

In Figure 5 (lower curve) we show the evolution of the total number of vaccine doses necessary as a function of the delay. The upper curves in Figure 5 give the evolution of the same quantity when we take into account the effect of vacations. Given the increase in the number of susceptible, a substantially increased vaccination rate is leading to a (perhaps exceedingly) high number of necessary doses. In fact, under such a scenario it is unclear whether the necessary vaccination rates are sustainable.

\subsection{Results for a partially efficient vaccine}

At this point we shall address the question of the consequences on a vaccination with a vaccine which does not confer a total immunity. A simple way to model this is the following. We assume that the vaccine has an efficiency $f$, smaller than 1 , and this results into a number of vaccinated individuals who can be infected. We can also assume that the fact that they are vaccinated attenuates somewhat the probability that they be infected, resulting in an infection rate smaller than the initial one. Introducing the population $T$ of persons who remain susceptible after having been vaccinated, and thus can be infected with an infection rate $b$, we obtain the following set of equations

$$
\begin{gathered}
\frac{d S}{d t}=-a S I-v \\
\frac{d T}{d t}=v f-b T I \\
\frac{d I}{d t}=a S I+b T I-\lambda I \\
\frac{d R}{d t}=\lambda I+v(1-f) .
\end{gathered}
$$

The infection rate $b$ can be smaller than $a$, meaning that the vaccination, although not totally effective, does confer some protection. If $b$ is equal to $a$, meaning no protection at all, then we can simply ignore the subgroup $T$ and work with the initial, single susceptible population, simply diminishing the vaccination rate by a factor $(1-f)$.

We investigate the effect of incomplete immunity conferred by the vaccine in the case where the vaccination is delayed. It is clear that if one does not compensate for the delay of vaccinations, the number of hospitalisations will increase with the delay.

This is indeed what is obtained by our model, as shown in Figure 6, where for the lowermost curve, we have roughly a factor of 2 between the number of persons hospitalised with a timely start of vaccinations and one delayed past the second epidemic wave. The continuous line corresponds to a $100 \%$ efficient vaccination, while the upper, dashed, line shows the impact on the number of hospitalisations of a vaccination campaign of efficiency 80 $\%$ where there is no protection offered by the $20 \%$ of inefficient vaccinations. In between the two extreme situations we find the results corresponding to cases where the vaccination, although not perfectly efficient, still offers some protection to vaccinated individuals. However, in all situations, when the delay is large, the impact of the partially efficient vaccines is inconsequential, while it is appreciable in the case of timely vaccinations. 


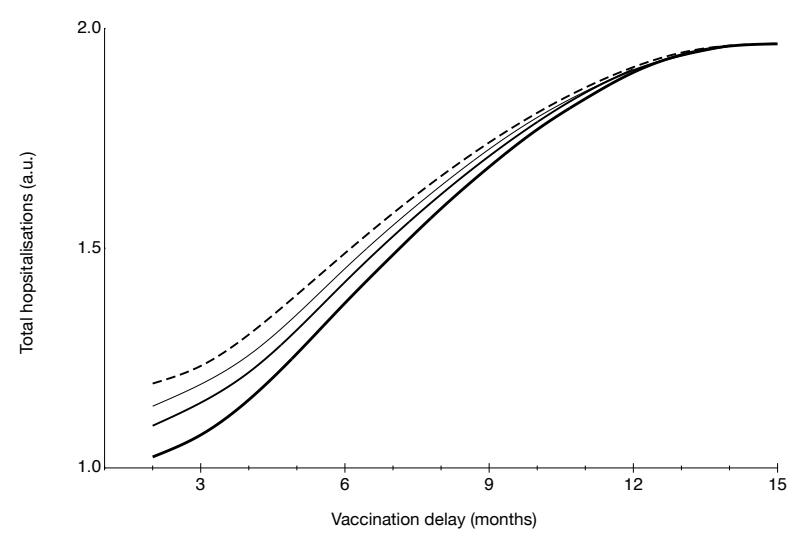

Figure 6. Total number of hospitalisations as a function of the vaccination delay, with a $100 \%$ efficient vaccination corresponding to $f=0$, (solid very thick lower line), a $80 \%$ efficient vaccination corresponding to $f=0.2$ and $b=0.5 a$, (solid thick line), a 80\% efficient vaccination corresponding to $f=0.2$ and $b=0.75 a$, (solid thin line) and a $80 \%$ efficient vaccination corresponding to $f=0.2$ and $b=a$, (dashed upper line).

The main conclusion from the elementary model we introduced in this section is that the timely implementation of vaccinations is of essence. The best scenario is when the vaccinations start with the appearance of the first wave of the epidemic, resulting in lower numbers of hospitalised patients (and consequently in a lower number of deaths). Similarly if one tries to compensate the initial delay of vaccinations by redoubling the vaccination effort, the price is a substantial increase in the total number of doses needed, to say nothing of the difficulties associated with an ever increasing vaccination rate.

\section{Results for a multi-component population}

In the previous section we investigated the effect of vaccination strategies on the number of persons needing hospitalisation and on the cost of vaccination delays. Our assumption there was that the population is constituted by a single group with the same characteristics as far as the infection is concerned. However it is clear, already from the outset of the epidemic, that the various age groups do not respond in the same way to the infection risk, the requirement of hospitalisation, let alone the probability of demise. In an extended study $\mathrm{Li}$ and collaborators [41] analysed the transmission patterns in Wuhan, in order to quantify the risks, concluding that it was the individuals older than 60 years which were at the highest risk of infection. Yu [42] performed a similar study, focusing on South Korea, reaching the same conclusion, namely that the risk for the elderly was higher and moreover significantly affected by the interaction with the other age groups. The CMMID COVID-19 group of the London School of Hygiene and Tropical Medicine [43] analysed epidemic data from a variety of countries, obtaining estimates of the susceptibility to infections. While they concluded that in countries with an overall younger population the relative incidence of clinical cases can be lower than in countries with older population, they point out that since the former are mainly low-income countries, it is possible that comorbidities do influence disease severity. Zhang et al [44] analysed the contact survey data from Wuhan and Shanghai, confirming what by now is widely accepted, namely that individuals of more than 65 years old are more susceptible to infection. 
Before investigating the effect of vaccination on an age-graded population, it is important to construct a model where the infection rates are properly fixed. If we assume that the total population is split into subgroups, instead of a universal infection rate $a$, we have a matrix $a_{i j}$, which corresponds to the rate with which the subgroup $j$ is infecting the subgroup $i$. In order to obtain this matrix we start from the contact matrix $c_{i j}$, which is the probability that members of the subgroup $i$ encounter members of the subgroup $j$ (which means that if the populations of the subgroups are equal, the matrix $c$ is a symmetrical one). In order to obtain the infection rates we must multiply each element of the contact matrix by the probability that, upon contact, a member of the subgroup $j$ can infect a member of the subgroup $i$ and vice versa. These two probabilities need not be equal and thus the resulting matrix $a_{i j}$ does not have to be symmetric even if all subgroups have equal populations. The resulting model has the form

$$
\begin{gathered}
\frac{d S_{i}}{d t}=-\sum_{j} a_{i j}(t) S_{i} I_{j}-v_{i} \\
\frac{d I_{i}}{d t}=\sum_{j} a_{j i}(t) S_{j} I_{i}-\lambda I_{i} \\
\frac{d R_{i}}{d t}=\lambda I_{i}+v_{i} .
\end{gathered}
$$

Again, the total population is conserved and we can introduce the normalisation $\sum_{i}\left(S_{i}+\right.$ $\left.I_{i}+R_{i}\right)=1$.

The seasonality applies to each element of the matrix: $a_{i j}(t)=a_{i j}(1.0+0.25 \cos (2 \pi t / T))$, with $T=12$ months. We define $R_{i j}(t)=a_{i j}(t) / \lambda$. The effect of vaccinations on an agegraded population has been studied by several authors. Moghadas and collaborators [45] studied the impact of vaccination in the USA and concluded that even with $95 \%$ vaccine efficiency the future epidemic outbreaks and resulting hospitalisations and deaths could be mitigated, already if only adults were vaccinated. Bubar et al [46] addressed the question of prioritisation in the light of a relatively limited initial supply of vaccines. They concluded that, while prioritising the young, 20-49, adults' vaccination could lead to minimising the number of infections, the overall mortality is minimised when the vaccination is targeting adults over 60 years of age. A similar prioritisation study was conducted by Wang and collaborators [47]. They concluded that vaccination deployment strategies should take into account the particularities of each region. According to them, the allocation scheme should be based on local epidemiology, underlying population health, projections of available vaccine doses but also the existing vaccine-hesitancy. The work of Rodriguez et al [48] presents the argument that prioritising the vaccines to groups with the highest number of interactions can reduce substantially the fatalities, provided children are also included in the vaccination campaign. Without children vaccination, which is the common practice in the current epidemic, the differences they find between the various priority strategies are toned down and the essential mechanism of limitation of the number of fatalities is the self-protection and awareness exhibited by the over- 65 population. In a similar study, Monod and collaborators [49], reach a surprising conclusion, namely that targeting the adults aged 20 to 49 for vaccination "is an important consideration in halting resurgent epidemics and preventing COVID-19 attributable deaths". This divergence of conclusions 
of the various modelling approaches shows that it is essential to stay within an as simple as possible model (albeit without sacrificing realism) trying to extract robust conclusions.

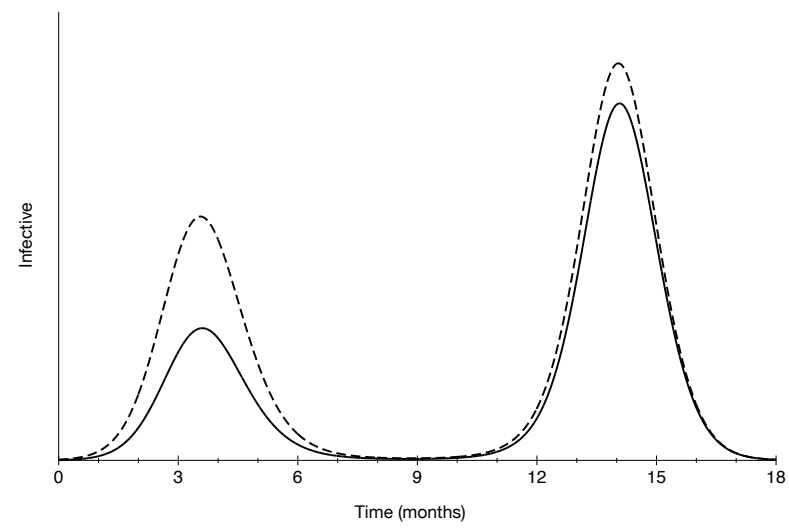

Figure 7. Temporal evolution of the infective fraction of the two age-groups, the "old" (solid line) and the "young" (dashed line), with neither vaccination nor recruitment.

We have performed several exploratory simulations involving two, three and four population subgroups. Our main remark is that increasing the number of subgroups increases disproportionally the number of parameters and thus the predictive potential of the model comes under question. So, unless one wishes to ask a very specific question (and, even in this case, the conflicting conclusions of the works cited above should act as a caveat) one should better stick to the simplest possible generalisation of the single-component population. Thus, in what follows, we shall present only results obtained in a two-component case. Our main assumption is that the total population can be split into two groups. One is a group consisting of the younger population up to roughly 60 years of age, the remaining comprising the older individuals. We decided that the "young" correspond to $70 \%$ of the total, while the "old" make up the remaining $30 \%$ (these proportions are realistic for most European countries). The young-old split allows one to answer in a more realistic way questions about the number of hospital admissions and/or deaths.

The infection rate matrix is difficult to estimate and is strongly dependent on the precise definition of the two age-groups and on the country demographics [50] . We thus chose an infection rate matrix with as realistic as possible values and which led (under the same seasonality assumption) to a two-wave epidemic, just as in the singlecomponent case, and with a proportion of young-to-old infected close to 2 in the first wave [51], see Figure 7. In the following, the index "o" will refer to the old group, whereas the index " $y$ " will refer to the young group. We choose the following values for the matrix: $R_{o o}=1.5, R_{y o}=1.0, R_{o y}=1.0, R_{y y}=1.2$, where $R_{y o}$ represents the number of infections among the "old" from one infective young person. The initial conditions are $I_{o}=I_{y}=2.0210^{-5}$.

The first question we shall ask is on the impact of the vaccination rate, of the older fraction of the population alone, on the number of hospitalisations (assuming that the "young" have 5 times less risk to be hospitalised while infected compared to the "old", $[52])$. 


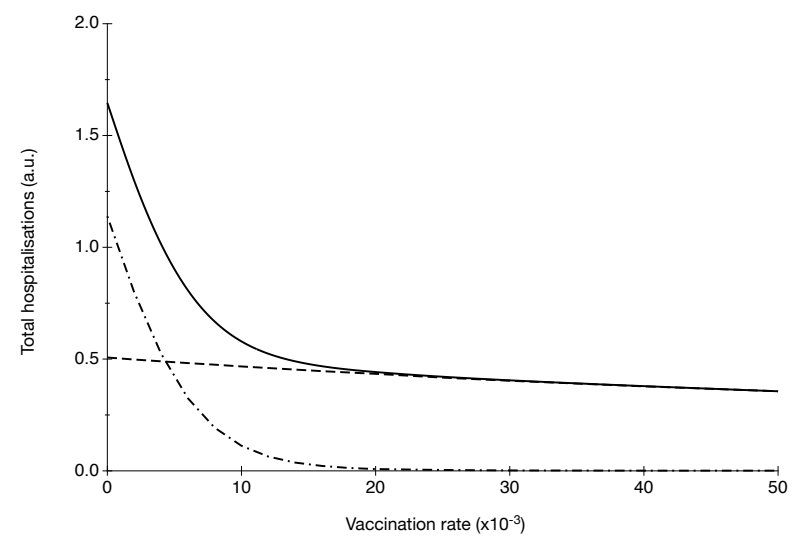

Figure 8. Number of hospitalisations as a function of the vaccination rate in the first wave, from $t=0$ to $t=7$ months, (dashed line), in the second wave, from $t=7$ months to the end of the simulation, (dashed-dotted line), and the total number of hospitalisations (solid line).

The results are shown in Figure 8. We have counted separately the number of hospitalisations for each of the waves (see Figure 7). Increasing the vaccination rate has a massive effect on the hospitalisations during the second wave, which go rapidly to zero. However the number of hospitalisations during the first wave diminishes but only very slowly. Thus it appears that it is not particularly interesting to increase excessively the vaccination rate. (In view of what we saw in the case of the single-component population and what we shall show below the crucial factor is the timely launching of the vaccination campaign).

Another question we can ask is what is the best balance between vaccinating the "young" and vaccinating the "old". Figure 9 provides a first answer to this. We started with a fixed vaccination rate and split it between the two components of the population going from 0 (only the old are vaccinated) to 1 (vaccination of the young alone). The result is clear: if one's aim is to minimise the number of hospitalisations the priority of vaccinations should be given to the older fraction of the population.

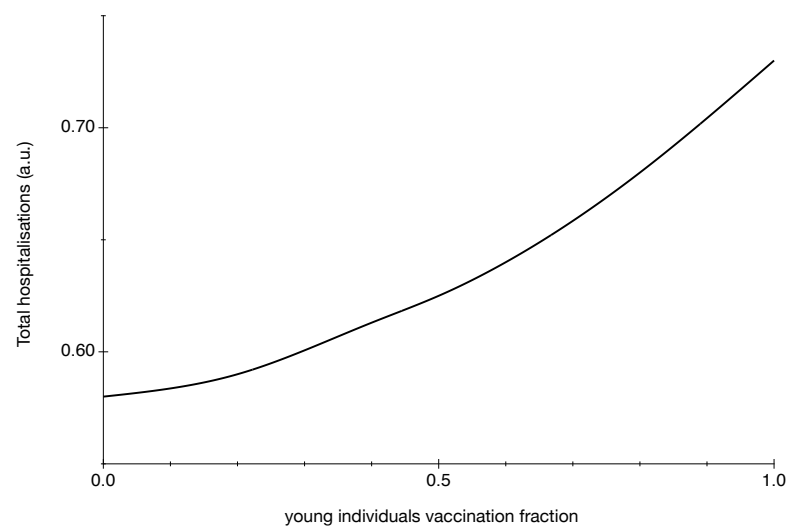

Figure 9. Number of hospitalisations as a function of the fraction of young people getting vaccinated. The total vaccination rate is set to $v / \lambda=10^{-3}$, and is split between the "old" and the "young" with varying proportions. 
A similar conclusion is reached when one studies the impact of the vaccination rate under the assumption that one vaccinates only the old or only the young (as well as any intermediate scenario).

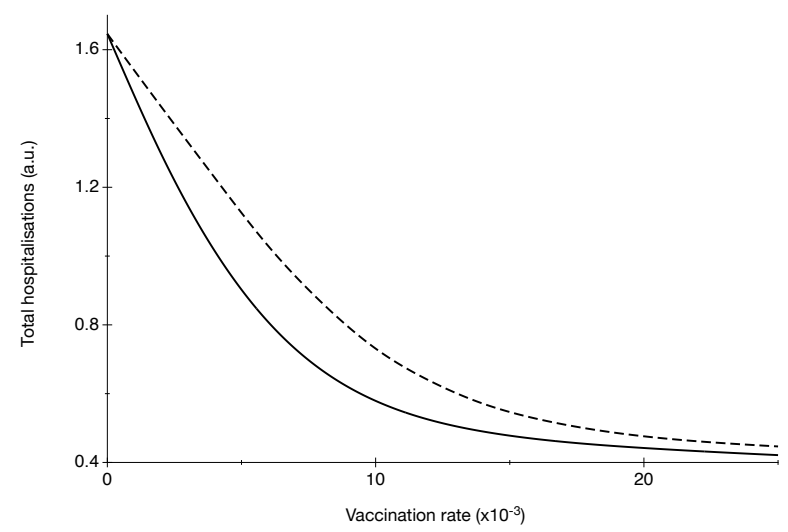

Figure 10. Number of hospitalisations as a function of the vaccination rate, when only old (solid line) or young (dashed line) people are vaccinated.

The number of hospitalisations is always smaller when one prioritises the old, although the difference can be toned down provided one is ready to vaccinate the young with a somewhat increased rate (see Figure 10). Moreover when the vaccination rate becomes really high it is not so important what fraction of the population gets vaccinated: the vaccinations do offer a global protection.

Given that the vaccination of the older fraction of the population plays a crucial part in diminishing the number of hospital admissions, in what follows we assume that the "old" get vaccinated at a fixed rate and as soon as the epidemic sets in.

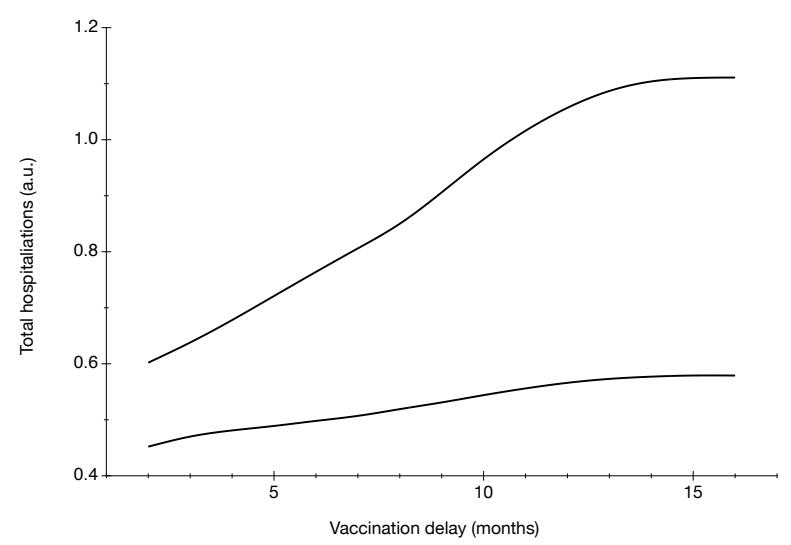

Figure 11. Number of hospitalisations as a function of the delay of the beginning of the vaccination campaign for the young people, without (lower curve) and with recruitment (upper curve). The vaccination of the old people starts at $t=2$ months and $v_{o} / \lambda=v_{y} / \lambda=$ $10^{-3}$. For recruitment $\mu_{0} / \lambda=510^{-4}$.

On the other hand the younger part of the population gets vaccinated with the same rate but at some later point. In Figure 11 we show the impact of this vaccination delay on the number of hospitalisations. The upper curve in Figure 11 represents the effect of a 
recruitment of susceptible population during the local summer vacation period. It is clear that a substantial delay combined with the recruitment effect can result into a (more than a) doubling of the number of hospitalisations. Again, the only recommendation in this case is to launch the young vaccination campaign the soonest possible since preventing the summer recruitment through restrictive measures is socially untenable.

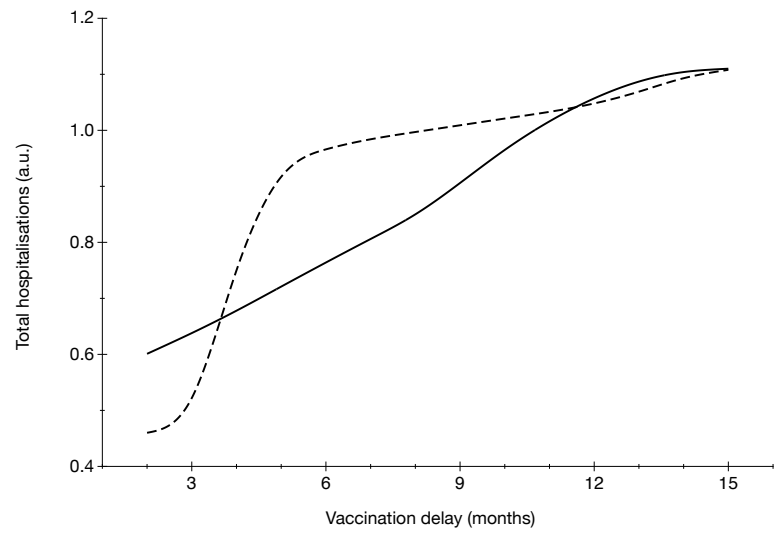

Figure 12. Number of hospitalisations as a function of the delay of the beginning of the vaccination campaign in the presence of recruitment, with fixed vaccination rate (solid curve) and with awareness-influenced vaccination rate (dashed curve) for the young people. The vaccination of the old people starts at $t=2$ months and progresses at a constant rate $v_{o} / \lambda=10^{-3}$. The rate of vaccination of the young people is not fixed and is proportional to the instantaneous number of infected. For each value of the delay, the coefficient of proportionality is chosen in order to use the same total number of vaccines as in the fixed-rate scenario. For recruitment, $\mu_{0} / \lambda=510^{-4}$.

The results of a final simulation we did perform are shown in Figure 12.

In this case we implemented what could be interpreted as vaccination-awareness. While we considered that the vaccination of the old was pursued unhindered, we assumed that the vaccination rate of the young was directly related to the number of infected individuals, increasing or decreasing with the number of infective. Practically this means that during the summer months, when due to the seasonality the epidemic ebbs and the number of infective diminishes, there are relative few among the younger population who think about getting vaccinated. In order to put this awareness-mimicking strategy into perspective we compare it with the results of a constant rate vaccination assuming that the launch of the vaccination campaign is delayed (see Figure 12). We fix the awareness-induced vaccination rate so as to have the same total number of vaccines used as in the fixed-rate scenario. The effect of summer vacation recruitment is taken into account in both cases. We remark that while awareness in a timely launched vaccination campaign can be interesting, once the vaccination is somewhat delayed it is more advantageous to proceed to vaccination at a fixed rate. For very long delays there is no difference between the two strategies since by this time it is too late for the vaccination to have any effect. 


\section{Conclusion}

In this paper we focused on the most important pharmaceutical intervention for the prevention of COVID-19, namely mass vaccination. Our aim was to study the consequences of the implementations of vaccine inoculation on a large population. Moreover, we interested ourselves in possible prioritisation effects and the possibility of optimising the vaccination strategy [53]. Our approach was based on the classic SIR model, justifying this choice over other (much) more complicated ones. Working with a simple model is more than sufficient when one aims at reproducing just the robust features of the epidemic evolution. In the current situation, where the ever-changing measures of the various governments influence the run of the epidemic, it is vital to focus on the most salient features, rather than aiming at reproducing details with dubious origins and usefulness.

Two main effects were the premises of our modelling approach: seasonality and recruitment. The first refers to modification of the infection rate due to climate. The second is meant to palliate for the lack of spatial component in the SIR model. In the present setting it encodes the increase in the number of susceptible individuals due to the population movement linked with the vacation period. Our model was tailored so as to lead to a multi- (minimally two-) wave epidemic. Introducing vaccination, we asked the question of what is its effect on the number of hospital admissions. (Given the close relation of the latter to the number of deaths, counting hospitalisations gives a good estimate of the death toll of the pandemic).

One of the assumptions used in most (but not all) of the simulations presented, was that the vaccines are $100 \%$ effective. This is not unreasonable as a first approximation but it is clear that one cannot expect all vaccines to provide an almost perfect immunity. It was thus essential that we study also the case of incomplete immunity, a feature which was easily incorporated into our model.

The main conclusion of this paper is that the timely launch of the vaccination campaign is of the utmost importance. Any delay results in an increase of hospitalisations and if one tries to compensate fo the delay by increasing the vaccination rate, this leads to a disproportionate expenditure of vaccination doses (and, perhaps, to unsustainable vaccination rates). Introducing a multi-age-group population we studied the effect of prioritisation of the vaccinations. The results in this case were indisputable: it is always advantageous to vaccinate the older population. Keeping this choice fixed, we asked the question of whether an awareness-based strategy for the younger population could be preferable. The implementation of "awareness" in this case was by adjusting (for the population in question) the vaccination willingness to the number of declared infections. It turned out that this strategy, was less efficient than the standard, constant vaccination rate, one, essentially due to the ebbing of the epidemic during the summer months.

The analysis presented in this paper raises several questions. For instance, is it possible to predict the end of the pandemic based on simulations? The answer to this question is unsurprising: with a global vaccination campaign and very efficient vaccines, it seems possible to eradicate the pandemic [54] reaching herd immunity. In the worst case scenario, COVID-19 would become endemic [55], recurring annually during the winter period. And while the next question lies beyond the scope of modelling, one can ask whether, in the case of a persisting, endemic, situation, a return to a "normal" style of living is possible. We believe that this is indeed possible provided measures are taken to facilitate the mobility of 
the fraction of the population which has acquired immunity to the virus [56]. Simulating the circulation of the susceptible, infective and immune population can, in principle, be contemplated in the framework of a multi-node SIR model. This model would constitute a definite improvement upon the naïve recruitment approach we presented here. It will be the object of some future work of ours.

\section{References}

[1] E. Landau, The Hard Lessons of Modeling the Coronavirus Pandemic, online at https://www.quantamagazine.org/the-hard-lessons-of-modeling-the-coronavirus-pandemic$20210128 /$.

[2] N. Perra, Non-pharmaceutical interventions during the COVID-19 pandemic: A review, to appear in Phys. Rep., https://doi.org/10.1016/j.physrep.2021.02.001

[3] C. Cheng et al., COVID-19 Government Response Event Dataset, Nature Human Behaviour 4 (2020), 756.

[4] T. Hale et al., A global panel database of pandemic policies, Nature Human Behaviour 5 (2021) 529.

[5] J. Brauner et al., Inferring the effectiveness of government interventions against COVID-19, Science 371 (2021) 802.

[6] European Centre for Disease Prevention and Control Guidelines for non-pharmaceutical interventions to reduce the impact of COVID-19 in the EU/EEA and the UK 24 September 2020. online at https://www.ecdc.europa.eu/en/publications-data/covid19-guidelines-non-pharmaceutical-interventions.

[7] G. Nakamura, B. Grammaticos and M. Badoual, Confinement strategies in a simple SIR model, Reg. Chao. Dyn. 25 (2020) 500.

[8] A.S. Fokas J. Cuevas-Maraver and P.G. Kevrekidis, Easing COVID-19 lockdown measures while protecting the older restricts the deaths to the level of the full lockdown, Scientific Reports 11 (2021) 5839.

[9] D. Bernoulli, Essai d'une nouvelle analyse de la mortalité causée par la petite vérole, et des avantages de l'inoculation pour la prévenir, Hist. et Mém. de l'Acad. Roy. Sci. de Paris (1766) 1.

[10] Z. Feng, S. Towers, and Y. Yang, Modeling the Effects of Vaccination and Treatment on Pandemic Influenza, The AAPS Journal 13 (2011) 427.

[11] P. Widyaningsih, A.A. Nugrubo, D.R.S. Saputro and Sutanto, Tuberculosis transmission with relapse in Indonesia: susceptible vaccinated infected recovered model, J. Phys.: Conf. Ser. 1217 (2019) 012071.

[12] X. Li et al., Estimating the health impact of vaccination against ten pathogens in 98 low-income and middle-income countries from 2000 to 2030: a modelling study, Lancet 397 (2021) 398. 
[13] M. Vossey et al., Safety and efficacy of the ChAdOx1 nCoV-19 vaccine (AZD1222) against SARS-CoV-2: an interim analysis of four randomised controlled trials in Brazil, South Africa, and the UK, Lancet 397 (2021) 99.

[14] F. Polack et al., Safety and Efficacy of the BNT162b2 mRNA Covid-19 Vaccine, N. Engl. J. Med. 383 (2020) 2603.

[15] G. Chodick et al., The effectiveness of the first dose of vaccine in reducing sarscov-2 infection 13-24 days after immunization: real-world evidence, preprint 2021, https://doi.org/10.1101/2021.01.27.21250612

[16] P.R. Hunter and J.S. Brainard, Estimating the effectiveness of the Pfizer covid19 BNT162b2 vaccine after a single dose: a reanalysis of a study of "real world" vaccination outcomes from Israel, preprint 2021, Medrxiv 2021.02.01.21250957v1.

[17] E. Mahase, Covid-19: Israel sees new infections plummet following vaccinations, BMJ 372 (2021) n338.

[18] COVID-19 Vaccine Breakthrough Infections Reported to CDC - United States, January 1-April 30, 2021. MMWR Morb Mortal Wkly Rep. ePub: 25 May 2021. DOI: http://dx.doi.org/10.15585/mmwr.mm7021e3.

[19] W.O. Kermack, A.G. McKendrick, Contributions to the mathematical theory of epidemics, Proc. Roy. Soc. Edinburgh A 115 (1927) 700.

[20] R. Ross, An application of the theory of probabilities to the study of a priori pathometry, Proc. R. Soc. Lond. A 92 (1916) 204.

[21] G. Nakamura, B. Grammaticos and M. Badoual, Recruitment effects on the evolution of epidemics in a simple SIR model, to appear in Reg. Chao. Dyn.

[22] A. Aleta and Y. Moreno, Evaluation of the potential incidence of COVID-19 and effectiveness of containment measures in Spain: a data-driven approach, BMC Medicine $18(2020) 157$.

[23] W. Roda, M. Varughese, D. Han and M. Li, Why is it difficult to accurately predict the COVID-19 epidemic?, Infectious Disease Modelling 5 (2020) 271.

[24] C. Merow and M. Urban, Seasonality and uncertainty in global COVID-19 growth rates, PNAS 117 (2020) 27456.

[25] T. Carleton et al., Evidence for ultraviolet radiation decreasing COVID-19 growth rates: global estimates and seasonal implications, Proc. Natl. Acad. Sci. USA 118 (2021) e2012370118.

[26] M. Sajadi et al., Temperature, humidity, and latitude analysis to predict potential spread and seasonality for COVID-19, JAMA Netw Open 3 (2020) e2011834.

[27] M. Watanabe, Early detection of seasonality and second-wave prediction in the COVID-19 pandemic, preprint 2020, https://doi.org/10.1101/2020.09.02.20187203 
[28] J. Dan et al., Immunological memory to SARS-CoV-2 assessed for up to 8 months after infection, Sience 371 (2021) 587.

[29] C. Gaebler et al., Evolution of antibody immunity to SARS-CoV-2, Nature 591 (2021) 639.

[30] W. K. Wong, F. Juwono and T. Chua, SIR Simulation of COVID-19 Pandemic in Malaysia: Will the Vaccination Program be Effective?, preprint 2021. arXiv:2101.07494v1 [physics.soc-ph] 19 Jan 2021.

[31] M. Schwarzinger et al., COVID-19 vaccine hesitancy in a representative working-age population in France: a survey experiment based on vaccine characteristics, Lancet Public Health 6 (2021) e210.

[32] B. Grammaticos, R. Willox and J. Satsuma, Revisiting the Human and Nature Dynamics model, Reg. Chao. Dyn. 25 (2020) 178.

[33] B. Grammaticos, A. Ramani, J. Satsuma, R. Willox, Discretising the Painlevé equations a la Hirota-Mickens, J. Math. Phys. 53 (2012) 023506.

[34] R.E. Mickens, Exact solutions to a finite-difference model of a nonlinear reactionadvection equation: Implications for numerical analysis, Numer. Methods Partial Diff. Eq. 5 (1989) 313.

[35] H. Hethcote, The Mathematics of Infectious Diseases, SIAM Review 42 (2000) 599.

[36] W. Shi et al., An extended Weight Kernel Density Estimation model forecasts COVID-19 onset risk and identifies spatiotemporal variations of lockdown effects in China, Communications Biology 4 (2021) 126.

[37] T. Menkir et al., Estimating internationally imported cases during the early COVID19 pandemic, Nature Communications 12 (2021) 31.

[38] M. Saito et al., Extension and verification of the SEIR model on the 2009 influenza A (H1N1) pandemic in Japan, Mathematical Biosciences 246 (2013) 47.

[39] Y. Zhao et al., Impact of population recruitment on the HIV epidemics and the effectiveness of HIV prevention interventions, Bull. Math. Biol. 78 (2016) 2057.

[40] I. Cooper, A. Mondal and C. Antonopoulos, A SIR model assumption for the spread of COVID-19 in different communities, Chaos, Solitons and Fractals 139 (2020) 110057 .

[41] F. Li et al., Household transmission of SARS-CoV-2 and risk factors for susceptibility and infectivity in Wuhan: a retrospective observational study, Lancet Infect. Dis. 21 (2021) 617.

[42] X. Yu, Risk Interactions of Coronavirus Infection across Age Groups after the Peak of COVID-19 Epidemic, Int. J. Environ. Res. Public Health 17 (2020) 5246.

[43] N. Davies et al., Age-dependent effects in the transmission and control of COVID-19 epidemics, Nature Madicine 26(2020) 1205. 
[44] J. Zhang et al., Changes in contact patterns shape the dynamics of the COVID-19 outbreak in China, Science 368 (2020) 1481.

[45] S. Moghadas et al., The impact of vaccination on COVID-19 outbreaks in the United States, preprint 2020, https://dx.doi.org/10.1101/2020.11.27.20240051

[46] K. Bubar et al., Model-informed COVID-19 vaccine prioritization strategies by age and serostatus Science 371 (2021) 916.

[47] W. Wang et al., Global, regional, and national estimates of target population sizes for covid-19 vaccination: descriptive study BMJ 371 (2020) m4704.

[48] J. Rodriguez, M. Patón and J. Acuna, COVID-19 vaccination rate and protection attitudes can determine the best prioritisation strategy to reduce fatalities preprint 2021, https://doi.org/10.1101/2020.10.12.20211094.

[49] M. Monod et al., Age groups that sustain resurging COVID-19 epidemics in the United States, Science 371 (2021) eabe8372.

[50] L. Shafer, M. Nesca and R. Balshaw, Relaxation of social distancing restrictions: Model estimated impact on COVID-19 epidemic in Manitoba, Canada, PLoS ONE 16 (2021) e0244537.

[51] N. Hozé N et al., Monitoring the proportion of the population infected by SARSCoV-2 using age-stratified hospitalisation and serological data: a modelling study, Lancet Public Health (2021), DOI:https://doi.org/10.1016/S2468-2667(21)00064-5.

[52] the CDC website: https://www.cdc.gov/coronavirus/2019-ncov/covid-data/investigationsdiscovery/hospitalization-death-by-age.html, April 27, 2021.

[53] M. Fitzpatrick and A. Galvani, Optimizing age-specific vaccination Science 371 (2021) 890 .

[54] S. Charunmilind, When will the COVID-19 pandemic end?, online at https://www.mckinsey.com/industries/healthcare-systems-and-services/ourinsights/when-will-the-covid-19-pandemic-end.

[55] J. Lavine, O. Bjornstad, and R. Antia, Immunological characteristics govern the transition of COVID-19 to endemicity, Science 371 (2021) 741.

[56] R. Brown, D. Kelly, D. Wilkinson and J. Savulescu The scientific and ethical feasibility of immunity passports, Lancet Infect. Dis. 21 (2021) E58. 